\title{
Advanced interaction media in nuclear power plant control rooms
}

\author{
Lucas Stephane \\ Human Centered Design Institute and Department of Mathematical Sciences, Florida Institute of Technology \\ 150 West University Boulevard, 32901 Melbourne, FL, USA
}

\begin{abstract}
The shift from analog to digital Instruments (related mainly to information visualization) and Controls in Nuclear Power Plant Main Control Rooms (NPP MCR) is a central current topic of investigation. In NPP MCR, digitalization was implemented gradually, analog and digital systems still coexisting for the two main systems related to safety - Safety Instruments and Control System (SICS) and Process Instruments and Controls System (PICS). My ongoing research focuses on the introduction of Advanced Interaction Media (AIM) such as stereoscopic 3D visualization and multi-touch surfaces in control rooms. This paper proposes a Safety-Centric approach for gathering the Design Rationale needed in the specification of such novel AIM concepts as well as their evaluation through user tests. Beyond methodological research, the final output of the current research is to build an experimental simulator aiming to enhance improvements in Human-Systems Integration (HSI). This paper provides an overview of the topics under consideration.
\end{abstract}

Keywords: Safety Centric Design, Human-Machine Interaction, process modeling, eye-tracking, situation awareness, workload, satisfaction

\section{Introduction}

The current research provides an overview of the activities carried out for enabling design and evaluation of potential novel design options that could possibly be integrated in the future in Nuclear Power Plant Main Control Rooms (NPP MCR).

It also aims to provide the structure and staging of the overall research, presenting the roadmap and rationale of the addressed topics.

Safety methods are mandatory for analyzing and understanding the Fukushima accident [12] that is the basis for the experimental scenarios.

At the same time, Safety methods encompass the Human Centric approach (i.e. design for safety [2]) and unify the goals of all other methods and technologies commonly proposed for Design and Evaluation (i.e. the Eye Tracking method is intended to explicitly contribute to the overall sociotechnical system safety).

The overall work performed within the scope of this research is to exploring safety related novel concepts for Instruments and Controls (I\&C) in the NPP MCR.
Thus, Safety principles are the kernel of the ongoing research. They guide Top-Down the methods, techniques and technologies utilized for the Design stage and Evaluation stage. These specific methods are consequently aligned on the overall Safety philosophy.

The current research tackles Safety on two main interrelated perspectives situated at the interaction frontier between humans and systems.

The first perspective is Visual Intelligence that expresses both human abilities to construct meaning from their visual environment and the ability of sociotechnical systems to deliver purposeful visual information.

The second perspective is Process Control that expresses the task-related contexts to which Visual Intelligence is applied as well as the means to control various processes that occur in such contexts.

The main problem statement, i.e. How to balance novel Advanced Interaction Media [3] (with a main focus on stereoscopic $3 D$ and multi-touch direct interaction) with existing ones (i.e. $2 D$ and indirect interaction) for ensuring safe equilibrium in operations 
through maximization of safety margins has to be decomposed into several subsequent questions.

Thus, in the following sections, the ongoing safety centric approach aims to deal with the following necessary questions:

- why novel design concepts are proposed in this research

- which Safety models and methods are relevant and how are they applied in the current research

- which specific Safety approaches enable to determine the consistency and usefulness of the proposed novel design concepts

- which are these novel concepts and their specification and implementation format(s)

- how Eye Tracking analysis may contribute in particular to verify and validate that the overall configuration of the visual environment (with a particular interest on the novel stereoscopic 3D I\&C) ensures a safe operational equilibrium

\section{Design Stage overview}

Safety principles and related approaches and methods are considered in the Design stage from a Design Rationale (DR) perspective.

In order to better identify possible design options, the first step in the current DR is to understand and analyze the Fukushima Daiichi accident (retrospective event analysis) as it occurred with the existing sociotechnical configuration at that time. The objective of this analysis is to identify specific safety requirements related to design flaws or lacks of upgrades of the NPP and related Instruments and Controls (I\&C) in the MCR.

This first set of requirements is then completed in a second step with safety guidelines applied to the novel design concepts proposed in the current research. These novel design concepts are split as related to:

1. the presentation of $I \& C$ in the MCR (i.e. requirements implementation)

2. the Design methodology itself (i.e. requirements management)

Even though the current research focuses on the first category, it is mandatory to consider the second category for two reasons. The first one deals with how the captured requirements are then implemented and instanced. The second reason comes mainly from the fact that with current integrated methods such as
Business Process Modeling \& Notation (BPMN [1, 22]) design and implementation become inseparable with a main focus on user requirements (i.e. intrinsically Human-Centric). BPMN integrated approach explicitly takes into account governance (and related breakdown, i.e. strategy and tactics) and provide architectural, structural and dynamic support for the Multi-Agent based Cognitive Architectures that are employed in the current research.

Based on the proposed split, novel design concepts are categorized as follows:

1. related to the presentation of $I \& C$ in the MCR:

a. introducing stereoscopic 3D representations for both $\mathrm{I} \& \mathrm{C}$ in the MCR

b. introducing direct interaction devices (i.e. multi-touch surfaces) in the MCR

c. introducing mobile handheld devices (i.e. tablets) in the NPP

d. introducing Scientific Visualization for presenting possible consequences of operational choices, mainly in the MCR

e. introducing sociotechnical system dynamics based on BPM/BPMN

2. related to the Design methodology

a. introducing BPM/BPMN for Human System Integration

b. transferring and refining the sociotechnical Multi-Agent based Cognitive modeling (both cool and hot cognition, i.e. rationality and emotions) from previous aeronautic studies to the current research in the NPP MCR

c. introducing the concept of loss-aversion as a central feature of the DecisionMaking process

d. introducing behavior modeling for reducing the discrepancies between prescribed tasks and real activities (ex ante and ex post)

The output of the Design stage is composed of:

- the sociotechnical model in terms of governance, architecture, structure and dynamics at the scale of the experimental lab

- design for safety requirements translated into specifications of the I\&C design concepts as well as their relevant combinations

- the implementation of selected design concepts in the experimental simulator 
In the current work, the overall safety approach spans and is related to situation awareness, selfawareness (i.e. emotions), workload and usability. Visual Intelligence features are emphasized and optimized for ensuring safe process control in the highly demanding visual environments, especially under accidental constraints.

The main purpose of the current Design stage is to identify and reinforce ex ante the optimal balancing between novel and existing design options. The safety centric approach is intended to provide operational positive equilibrium through the maximization of safety margins.

\section{Evaluation stage overview}

At this stage, the design options specified and implemented in the second design step are tested experimentally with users.

It is important to mention at this point that since the access to real NPP operators is not sure, the evaluation methodology was extended to enable experiments with users who do not belong to the nuclear domain. Such experiments enable to provide out of the box considerations both on the information presentation layer (visualization and interactivity) and on the decision making process itself, since they are neutral concerning either saving the plant or preserving the environment.

For this reason, the experimentation uses RolePlaying Game (RPG) paradigms.

This extended methodology could be as well performed with NPP experts when opportunities to access such experts will occur in the future.

The experimental scenario is based on the Fukushima Daiichi accident. Choosing this accident scenario enables to address mostly critical features of the proposed design options. Furthermore, it enables a better immersion and involvement of users in the experiment. In this accidental scenario, safety is assessed based on the ability of the sociotechnical NPP system to:

1. provide the optimal support for organizational and operational decision making in the MCR; in the current research, even though frontline operators and MCR I\&C are the main focus, governance in terms of safety culture and related breakdowns have to be seriously considered

2. shutdown the reactor

3. maintain after shutdown the required configuration in terms of radioactivity, depending on fuel, temperature and pressure, both for the reactor and spent fuel storage pool

4. provide successful escalation alternatives (resilience) if the required configuration fails

In the scope of the current research, the main focus is on the first point, but the other points are intertwined in terms of outcomes of decision chains and their repercussions in terms of contextual changes, i.e. NPP states represented in the NPP MCR. It is also important to notice that the escalation alternatives that may be designed (i.e. resilience) depend completely on the plant governance that defines the thresholds between the various steps as well as the related decisions. For example, assuming that the NPP provides all possible features related to escalation (including containment in all possible NPP states), the main question is at which threshold the attempts to save the NPP are stopped and the decision to preserve the environment occurs (i.e. goal change)? Such considerations are tightly linked with the concept of safety margins mentioned earlier.

The experimental design implements such possible alternatives expressing them explicitly as spatiotemporal losses in terms of living beings (including humans), systems and environment. The spatial facet expresses the proximity or the distance of the consequences as well as the area of the spreading. The temporal facet expresses the time frame of the consequences, from immediate to long lasting effects (i.e. several hundreds of years).

Various configurations of the experimental simulator support the main experiments related to NPP MCR. These configurations are based on various integrated combinations of design options, i.e. combinations of current 2D instruments and representations - i.e. Micro Simulation PC Tran for the reactor, Spent Fuel Pool (SFP) and Puff for dose dispersion and related physical controls and novel stereoscopic 3D instruments, scientific visualization (which may be either 2D or 3D) and related touch-based virtual controls. Previous to (or in parallel with) these main experiments, individual design options should be tested from a pure usability perspective. These usability tests provide general feedback on the stereoscopic 3D I\&C, touch based interaction and scientific visualization. Feedback from these usability experiments will be iteratively taken into account for possible upgrades of the proposed design options.

Since the proposed I\&C are highly visual, the main evaluation methods are Eye Tracking [5] and Situation Awareness [6, 19]. The accidental context requires adding Self Awareness (i.e. emotions) as well as workload assessment methods. 
In particular, the results of Eye Tracking applied to stereoscopic 3D scenes are expected to tightly contribute to their verification and validation based on safety criteria. Previous experiments in a civil aircraft cockpit simulator where Eye Tracking was applied to a 3D Synthetic Vision System integrated with regular $2 \mathrm{D}$ instruments had valuable outputs in terms of cognitive structures (i.e. visual patterns $[11,18]$ ) gathered with the Eye Tracking method. The challenge of the current research is to expand Eye Tracking investigations in stereoscopic 3D environments related to NPP MCR. Specific outputs of Eye Tracking in such a context are crucial for determining the ability of such novel designs to improve safety.

\section{Safety considerations for the novel Design Options}

The novel design options proposed for the MCR are stereoscopic 3D I\&C, scientific visualization and multi-touch surfaces. These novel design options may be combined among themselves and integrated with existing $2 \mathrm{D}$ instruments and physical controls. The motivation of proposing these specific novel design options is provided below.

\subsection{Stereoscopic $3 D I \& C$ for direct visualization}

Current 2D visual environments are abstractions of the real world. Such 2D representations must always find ways to deal with the missing third dimension that is part of the real world. For example, in aircraft cockpits the real 3D world is represented with two 2D planes on the main cockpit instruments, i.e. a frontal 2D view on the Primary Flight Display (PFD) and a horizontal view from above on the Navigation Display (ND). These two views are augmented with various features representing the necessary flight parameters as well with several possible visualization modes for the ND.

Based on these 2D representations, the human operator (pilot) has to build the 3D model of the real situation.

The effects of such 2D representations are not context dependent and thus generalizable. While gathering information on 2D instruments, operators have to spend cognitive energy to build or adjust their situational mental models.

In the current research, stereoscopic 3D representations are mostly abstract, i.e. they are not primarily intended for representing high fidelity systems of the plant. They are proposed primarily to improve operators' mental models and thus to minimize when possible the steps and cognitive energy for building and adjusting mental models based on 2D representations. This approach is guided by the direct visualization of necessary plant parameters.

Furthermore, stereoscopic 3D representations enable to provide a more homogeneous and compact visual environment compared to the current MCR configuration where information is spread on a large number of panels and displays.

Beyond visual representations of information (i.e. instruments), controls can also be embedded and displayed in the stereoscopic 3D scene. Such controls are not necessarily representations of existing physical artifacts. Controls can be either 2D or stereo 3D. The interaction with the visual environment can thus be supplemented by such controls implementing direct interaction (see section below), or performed with physical controls or mixed.

It is worth to notice that touch surfaces are physical devices. However the control itself is virtual. In this text, a clear distinction is made between the terms digital and virtual. For example, a computer keyboard is digital but a keyboard displayed on a device (i.e. display, phone, tablet) is considered as virtual.

\subsection{Scientific Visualization for assessing possible consequences}

Scientific visualization is related to the analytic representation of information. It emerged in the $70 \mathrm{~s}$ and focuses on the meaning conveyed by statistical analysis presentations [20]. This initial initiative was extended and is currently critical for large-scale analytical systems. In such environments it is crucial to succeed in presenting vital information in the right format in order to convey meaning for decision support $[7,20]$.

In the current research, analytical information is proposed in the MCR for providing insights and anticipating the possible consequences of alternative actions (what-if scenarios). Such analytics and related visualizations may be quantitative (i.e. amplitude, spreading) as well as qualitative (i.e. safety margins dynamics).

They could be either 2D or stereo 3D. They could be displayed apart, embedded in the main visual environment or mixed. 


\subsection{Multi-touch surfaces for direct interaction}

The emergence of multi-touch surfaces is subtended by the direct interaction principle that increases natural interaction. Users can interact with the displayed information by touching and performing gestures on it. In the current research, two types of multi-touch surfaces are considered: fixed and mobile.

Fixed multi-touch surfaces are added to 3D stereoscopic displays. They enable thus possible interaction with stereoscopic 3D information. They are intended for being utilized in the MCR.

Mobile multi-touch surfaces are standalone handheld devices (i.e. tablets). Several use-cases are possible.

The first use-case enables to interact with the information displayed by these devices themselves that could be either 2D (in most cases) or 3D only (not stereo). In this case they are intended for mobile operators in the plant.

The second use-case enables to remotely interact with visual environments displayed on other devices (i.e. remote desktop, remote video cameras, virtual track pad, virtual keyboard, etc.). This case is also extending the embedded controls described previously.

\section{Relevant Safety models and methods applied in the current research}

\subsection{FRAM}

The Functional Resonance Accident Method (FRAM) [8] enables to systematically analyze how multiple functions and conditions interrelate and combine to produce adverse outcomes. FRAM is aligned on the principle of proactive safety (i.e. resilience) and thus belongs to the latest generation of safety methods going beyond traditional causeeffects links, i.e. structural approaches that backtrack from the outcome until a satisfying causal set is identified. FRAM considers current operations and focuses on their combined variability (functional resonance replaces linear causality). As such, FRAM overpasses for example the complex-linear Swiss cheese model (Reason).

FRAM is selected in the current research based on the modular decomposition and clear guidelines and steps it provides.

FRAM is subtended by 4 principles:
1. the principle of equivalence of successes and failures, i.e. successes express the ability of the overall sociotechnical system to cope with real world complexity, failures express the temporary or permanent inability to cope; both successes and failures are related to the overall design philosophy based on resilience

2. the principle of approximate adjustments of operations to real conditions that never totally match the initial specifications; since resources are finite, the adjustments are approximate rather than precise

3. the principle of emergence expressing the variability coming from multiple combined functions (in current sociotechnical systems the variability of only one function is generally never sufficient to cause accidents)

4. the principle of functional resonance expressing the dynamic impact of the variability of multiple combined functions and its nonlinear propagation

FRAM can be used either retrospectively (FRAM Accident Analysis or FRAM AA) or prospective (FRAM Risk Analysis or FRAM RA). In the context of the ongoing research, since the proposed design options are in an early stage, FRAM is intended to be utilized mainly retrospectively for analyzing the $\mathrm{Fu}-$ kushima accident.

The steps in FRAM AA are:

1. Identify essential system functions using 6 aspects:
a. Inputs (I)
b. Outputs $(\mathrm{O})$
c. Preconditions $(\mathrm{P})$
d. Resources $(\mathrm{R})$
e. Time constraints $(\mathrm{T})$
f. Controls (C)

2. Characterize the variability of each function (current and potential)

3. Identify and describe the functional resonance in terms of dependencies and couplings among functions

4. Identify barriers for variability
a. Physical
b. Functional
c. Symbolic (indications of constraints on action that are physically present)
d. Incorporeal (indications of constraints on action that are not physically present)


FRAM will be used for the global analysis of the Fukushima Daiichi accident since it enables to tackle negative outcomes (i.e. accidents) considering normal operations - in this case regular abnormal procedures were applied during the accident (the Daiichi reactor was shut down but the required conditions could not be ensured over time).

\subsection{ETTO}

Efficiency-Thoroughness Trade-Off Principle [9] is of particular interest for the novel design options proposed in the current study. The ETTO principle emphasizes the fact that in real situations, since resources are limited, operational trade-offs occur between the interrelated efficiency (i.e. necessity to act) and thoroughness (i.e. necessity to think and understand the current situation). In some cases fast action reduces initial understanding and too much resources allocated to understanding may postpone required actions.

The aim of the novel design concepts is to improve both efficiency and understanding. Consequently, ETTO will be used as a driving principle in design.

\subsection{STAMP, STPA and SpecTRM}

\subsubsection{Intent Specifications}

Intent Specifications [16] are improved sets of practices for requirement specifications and specifications. Intent Specifications were developed based on the observation that most accidents related to safety-critical systems were tracked back to issues at the requirements level. Intent Specifications are organized hierarchically, to answer why decisions were made, what is to be done and how can it be accomplished. This particular Design Rationale that captures designers' intentions is called Intent Specifications.

The structure of an Intent Specification spans the Environment, Operators, Systems and Components and related levels (1 to 6).

Verification and Validation (V\&V) specific steps are associated with each level.

Vertically, higher levels depend on lower levels.

Traceability is ensured between levels, from upper levels to lower levels (inter-level traceability), i.e. from specifications to design, implementation and operations. Furthermore, traceability is ensured intralevel.

\subsubsection{STAMP and STPA}

The specificity of Systems-Theoretic Accident Model (STAMP) is that it is not based on narrow event sequences, as traditional models do, but spans the whole sociotechnical system in terms of entities, combinations and interactions. Furthermore, all entities are considered in terms of their impact on the overall system safety. STAMP emphasizes constraints and constraint violation instead of only events. Safety is ensured as a dynamic equilibrium based on feedback loops of information and control. Risk Assessment is based on the reinforcement of constraints (i.e. safety margins).

STAMP To Prevent Accidents (STPA) [10] is a hazard analysis technique based on the STAMP model. Hazardous states are considered as the result of control issues due to various causes, including the sociotechnical system entities. The main focus is on dysfunctional interactions within the overall system as well as outside, i.e. manufacturer organization, regulatory bodies, governments, etc.

STPA has 5 steps:

1. identify system hazards

2. identify system-level safety-related requirements and constraints

3. define the basic system control structure

4. identify inadequate control actions that could lead to a hazardous system state

5. determine how the system safety constraints could be violated and minimize such possible violations

5a. create the process models for components under study

5b. analyze identified process sections and related controls in terms of possible issues

In order to improve safety, STAMP focuses on contextual control loop flaws and related decisionmaking, such as:

1. inadequate enforcement of constraints

2. inadequate execution of control action

3. inadequate or missing feedback

STPA enables to

- $\quad$ link entities as a network (each entity being a node),

- $\quad$ represent various system states

- represent the timeline

- check the specification completeness (based on attributes) 
- analyze possible mode confusion (mode transition)

STPA will be performed to complete the FRAM analysis of the Fukushima Daiichi accident. SpecTRM might be utilized to some extent for accomplishing this analysis.

\subsubsection{SpecTRM}

For safety critical systems, the Specification Toolkit and Requirements Methodology (SpecTRM) [16] implemented as a software enables to:

- create Intent Specifications

- perform STPA

- validate models

- run simulations

SpecTRM will be used for guiding the specifications of the novel design options as the main requirements management model and toolkit.

\section{Experimental Simulator of the NPP-MCR}

The set of identified requirements concerns:

- novel information visualization (stereoscopic 3D ) related to Instruments

- paradigms to represent large volumes of information (Scientific Visualization)

- multi-touch devices related to Controls, for improving direct manipulation of the graphical items

- processes and human workflows related to improve and accelerate information sharing and action for operators in the main control room and between operators in the main control room and distributed operators either within the plant (i.e. maintenance) or outside the plant.

The simulator specifications are thus related to a minimum and necessary set of subsystems that enable to represent subsets of plant complexity. However, in doing so it is not necessarily possible to limit the simulator to plant subsystems apart, since the goal of the experimental simulator is to capture relevant overall behaviors for the study, even though one of its main roles is to provide and test proofs of concept and their safety and usability related to the various topics under study.

As mentioned in the introduction, two main categories of topics have to be addressed with a Safety Centric model:

1. Information Visualization
2. The set of processes that feed the visual items displayed on the User Interface and that specify the various interaction modes

\subsubsection{Visualization and Interaction}

The main control room manages the main organs of the plant that are [15]:

- the reactor

- the spent fuel pool

- $\quad$ the cooling systems (primary \& secondary)

- the turbines

On one hand these organs can be further decomposed into sub-systems and components and on the other hand these organs have to be interrelated.

Paradigms utilized currently for displaying these systems, subsystems and components in the main control room are $2 \mathrm{D}$ representations that combine a $2 \mathrm{D}$ realistic representation of the system itself and additional diagrams and parameters.

Lessons learned show that such 2D representations have their benefits in terms of accuracy. However, when using their Mental Models [13], operators have to process several partial mental representations in order to retrieve the real model of the artifact.

Thus, a central goal of the usability simulator is to propose stereoscopic 3D representations when they can replace and overpass specific $2 \mathrm{D}$ representations. These 3D representations are intended to coexist with the previous $2 \mathrm{D}$ representations in a mixed configuration. The balance between both kinds of representations will be finally identified with user tests including eye tracking, situation awareness, satisfaction and workload methods.

Another topic addressed by the simulator is the visualization of large volumes of data. Current trends, methods and products already utilized in other domains could be valuable in main control rooms. Such approaches are based on digital dashboards [7], visual clusters and structures that are rooted in the human visual system capabilities and are mostly related to visual perception [21] and meaning conveyed by visual representations [20].

What-if or predictive tools that enable operators to simulate the consequences of their possible actions in advance are also introduced for improving operational decision-making.

Multi-touch surfaces are categorized as fixed (i.e. displays) and mobile (i.e. handheld tablets). For fixed multi-touch surfaces, visual instruments and controls are merged on the same device, introducing new interaction paradigms. Gestural interfaces [17] and interaction were already studied for $2 \mathrm{D}$ visualizations. 
The challenge of the ongoing research is to address the safety and usability of multi-touch applied to stereoscopic 3D visualizations. Mobile touch tablets provide remote desktop features that have also to be integrated with the stereoscopic $3 \mathrm{D}$ visualizations on the main remote systems.

\subsubsection{Processes}

In interactive environments, visual gathering of information is a trigger for decision making and action and conversely actions (that change the visual scene) are triggers for new iterations of visual gathering. Thus, the whole interaction process has to be modeled related to the various systems and information flows represented on the visual scene.

Central concepts in process modeling are $[1,22]$ :

- Orchestration (harmonization and tempo)

- Choreography (protocols and behaviors)

- Collaboration (interactions) ty)

- Escalation (resilience principles; proactive safe-

These concepts can be applied as well at low and high design levels. They are thus used for designing the novel interaction itself but also for higher levels of considerations in the NPP MCR such as social interaction within or outside the NPP - respectively between plant operators and with operators outside (i.e. rescuing organizations, etc.) Thus, such process modeling in the experimental lab will drive and support the safety-centric novel design options proposed.

\section{Conclusion and perspectives}

The diverse topics addressed in this paper constitute a basis of requirements for specifying the experimental simulator of the NPP-MCR. The Accidental scenario based on the Fukushima events is being setup for experimentation. The software pipeline [4] for implementing 3D stereoscopic representations as well as interactivity was identified and acquired. Devices such as the stereoscopic 3D display and projector, touch surface and tablets as well as the eyetracking system are integrated in the experimental lab. Experiment design is almost completed. The first series of experiments will start during Fall 2011.

In the near future, iterations in design and evaluations will enable to stabilize and refine the overall approach for the NPP-MCR novel design concepts from a safety-critical Human-System Integration perspective.

\section{References}

[1] Allweyer, T., BPMN 2.0: Introduction to the Standard of Business Process Modeling. Herstellung und Verlag, 2010

[2] Boy, G.A., Design for Safety: A Cognitive Engineering Approach. In Safety of Intelligent Driver Support Systems. Design, Evaluation and Social Perspectives. Ashgate, UK, 2011

[3] Boy, G.A. (1991). Advanced interaction media as a component of everyday life for the Coming Generation. Proceedings of the World Marketing Congress, Japan Management Association, Tokyo, Japan

[4] Creighton, R.H., Unity 3D Game Development by Example, Packt Publishing, 2010

[5] Duchowski, A.T., Eye Tracking Methodology: Theory and Practice. Springer Verlag London, 2003

[6] Endsley, M.R., Designing for situation awareness; An approach to User-Centered Design. Taylor \& Francis, London, 2003

[7] Few, S. Information Dashboard Design, O’Reilly, 2006

[8] Hollnagel, E., Pruchnicki, S., Woltjer, R. \& Etcher, S. Analysis of Comair flight 5191 with the functional resonance accident model. 8th International Symposium of the Australian Aviation Psychology Association. Sydney, Australia, 2008

[9] Hollnagel, E. The ETTO Principle: Efficiency Thoroughness Trade-off. Ashgate, 2009

[10]Leveson, N.G. Engineering a Safer World: Systems Thinking Applied to Safety. MIT Press, 2011

[11] Magnusson, S. M., Discovering hidden time patterns in behavior: T-patterns and their detection. Psychonomic Society: Behavior Research Methods, Instruments \& Computers 2000, 32 (I), p. 93-110, 2000

[12] Micro Simulation Technology, Fukushima Earthquake Station Blackout Event PCTran Analysis, 2011

[13] Ochanine V.A. Concept of operative image in engineering and général psychology, in B.F. Lomov, V.F. Rubakhin, and V.F. Venda (Eds). Engineering Psychology. Science Publisher : Moscow, 1977

[14]Pew, R.W., Mavor, A.S., Human-System Integration in the System Development Process. National Academies Press, pp. 36-90; 277-330, 2007

[15]Raja, A.K. et al., Power Plant Engineering. New Age International, 2006

[16] SpecTRM User Guide. Safeware Engineering, 2011

[17] Saffer, D., Designing Gestural Interfaces. O’Reilly, 2008

[18] Stephane, L. Visual Patterns in Civil Aircraft Cockpits. In Proceedings of the International Conference on HumanComputer Interaction in Aeronautics, September 20-22, Seattle WA USA, p. 208-214. Cépaduès-Editions, Toulouse, France, 2006

[19] Taylor, R.M., Finnie, S.E., MacLeod, I. Enhancing Situational Awareness Through System Cognitive Quality. Defence Research Agency, Farnborough, Hampshire, 1996

[20]Tufte, E.R., Envisioning Information, Graphics Press, 1990

[21] Ware, C., Information Visualization: Perception for Design. Morgan Kaufmann Academic Press, 2000

[22] White, S.A., Miers, D., BPMN Modeling and Reference Guide, 2008 